Volume 8, No.6, November - December 2019

International Journal of Advanced Trends in Computer Science and Engineering

Available Online at http://www.warse.org/IJATCSE/static/pdf/file/ijatcse104862019.pdf

https://doi.org/10.30534/ijatcse/2019/104862019

\title{
CBARG-Cultural Based Optimized Association Rule Generation Method to improve Knowledge Discovery in Autism Spectrum Disorder
}

\author{
R.Abitha ${ }^{1}$, Dr S. Mary Vennila ${ }^{2}$ \\ ${ }^{1}$ Research Scholar, PG \& Research Department of Computer Science, Presidency College, \\ University of Madras,Chennai,India,abirae2000@yahoo.co.in \\ ${ }^{2} \mathrm{Head}$, Associate Professor, PG \& Research Department of Computer Science, Presidency College, \\ University of Madras, Chennai, India, vennilarhymend@yhoo.co.in
}

\begin{abstract}
Association rule mining (ARM) is one of the most sought out techniques by the researchers as it intends to extract the association among the features in any data base. ARM makes significant amount of rules, which are hard for end clients to comprehend or approve, in this manner it confines the use of excavating results. Apriori algorithm is utilized commonly to generate the frequent item sets and extensive quantity of association rules. However The rules generated by Apriori algorithm is measured using support and confidence of minimum values and are not enough metrics to evaluate the strength of the rules generated. In this paper, another rule mining algorithm has proposed considering Cultural Algorithm (CA) called Cultural-based Association Rule Generation (CBARG) strategy, to reduce the quantity as well as to generate optimized association rules. It relies upon the likelihood that rules which are superfluous and not of high support have expelled from the information. It is perceived from the result obtained the proposed CBARG algorithm produce better result with other rule generation algorithms.
\end{abstract}

Key words : Association Rule Mining, Apriori Algorithm, Rule Generation, Cultural Algorithm, Optimization.

\section{INTRODUCTION}

In the recent decades real word database has rapid growth with massive amount of data. This enormous amount of data is available in different transactions fields like marketing, medical, networking, social media and oceanology and to name a few. Wide-range of techniques are existing in Machine learning to analyze and explore effectively the hidden and interesting patterns present in this huge data. Association rule mining (ARM) targets to extricate fascinating correlations, frequent examples, association structures among sets of attributes presented in the databases [3] .The primary point of ARM is to discover rules that fulfill predefined least support and confidence from a given database [5]. It works in two phases. In the first phase candidate item sets are generated that fulfills the predefined minimum support and confidence. In the second phase to produce association rules from enormous item sets with limitations of negligible confidence [7].

As a rule, ARM creates amazingly massive amount of rule, which are inconceivable for end clients to appreciate or approve, and restricting the utility of data mining results [14]. Various algorithms have been proposed to decrease the quantity of association rules [8] [9] [10], for example, creating just attractive rules or non-excess rules, or rules fulfilling certain criteria, for example, strength or lift, leverage and coverage [9]. The best method to decrease the quantity of rules to produce comprehensive knowledge with user interested properties is optimization of rules with evolutionary algorithm[22]. Still significant algorithms for controlling huge number of association rules are frequently computationally costly and potentially create a lot of unimportant rules. These issues can be handled by the proposed method Cultural algorithm based association rule generation (CBARG) Cultural Algorithm (CA)[17].

\section{AUTISM SPECTRUM DISORDER}

Autism is a neurological developmental disorder condition has the challenges social interaction, communication and cognitive behavior. Autism affect 1 in 110 children which is comparatively higher in the number than children affected with AIDS, cancer and diabetes combined. Autism is called Autism Spectrum Disorder (ASD) as the symptoms fall in to the broad spectrum of scale. In each adults and children, the signs and side effects of the autism spectrum disorders grasp issues with social association aptitudes, discourse and correspondence. The autism spectrum disorders are assessed dependent on the nearness of numerous side effects that disturb the kid's capacity to talk, cause the connections, to explore, play, and to ponder. The side effects of autism spectrum disorders: Social abilities. Essential social connection might be problematic for children with autism spectrum disorders.[12] Side effects may include: 
- Unusual or unseemly visual correspondence, motions, and outward appearances (for example maintaining a strategic distance from eye to eye connection or outward appearances that don't coordinate what the person is stating).

- Lack of enthusiasm for individuals or in sharing interests or accomplishments (for example demonstrating you a drawing, indicating a winged creature).

- Unlikely to move toward others or to seek after social collaboration; seems to be detached and held; likes to be separated from everyone else.

- Problem and trouble in understanding unique individual's emotions, responses, and nonverbal prompts.

- Resistance to being contacted.

- Difficulty or inability to make companions with children a similar age.

\section{RELATED WORKS}

There are stacks of literature used association rule mining to find solution to various problems in different domains. But to the best of our knowledge the proposed method in this paper CBARG is the first attempt for optimization of association rules. Jerry et al.[1] proposed high utility item set mining and measured the item set in terms of quantity and profit according to user preference. Fadi Thabtah etael [2] proposed a new rule induction algorithm that reduce the search space by the method of early pruning and made item frequency as dynamic one rather static which is helpful for the classifier to make decision from the minimal set of rules. The author has tested the algorithm with different data set from UCI repository and implemented in WEKA Martínez-Ballesteros et al.[4] proposed new representation for stated attributes in the rules are coded and presented for cross over operation rather coding all the attributes in the data set hence e the scalability of genetic algorithm for rule mining in large data set is enhanced. Thang Mai et al.[5] have built an algorithm for high utility association rules using lattice structure and proved with recommendation system that it takes less run time and less memory space. Uros Mlakar et al.[8] proposed a modified binary cuckoo search with novel binary representation of individuals and single objective optimization for association rule mining with modified cuckoo search and recorded promise able results with different UCI data. M.Sathya et el.[13]introduced Eclat and PSO based mining on association rule mining using éclat to improve sales in super market and proved with good accuracy and minimization of rule generation with optimization. Le Hoang Son et al, N.S. Nithya et al.[14][18] stated performance of the algorithm is degraded due to irrelevant rules and increased computational time by association rule mining for finding frequent rules. Muhammad Kamal Amjad et al.[16]presented in his survey that genetic algorithm is a popular technique for the solution of flexible job shop scheduling problems and stated hybrid GA is more popular than pure GA. Kargarfard et al.[19] presented a combination of classification and association rules to detect influenza disease which they trust can be lead to a decision and expert system. Maziyar Grami et al.[21] proposed modified GA performed well compared to GA, apriori and FP growth but time consumption is more compared to apriori and FP growth when tested on transactional database. Youcef Djenouri et al[22] presented improved BSO-ARM with three different heuristics that outperforms in terms of fitness and CPU time.Wang et al.,[23] introduced new fuzzy technique to analyze data set and discover association rules and sequence corrosion in chemical process Mohit K.Gupta et al.[26] proposed optimization of multi objective features of GA and achieved good performance with different UCI data sets.

\section{ASSOCIATION RULE MINING (ARM)}

An association rule mining is discovering the frequent patterns, relations, informal structures, interesting hidden patterns, correlations among the set of objects in the information repositories. In data mining, association rule mining is an accepted and well performing method for discovering motivating relations between the variable and target class in large databases. It is proposed to identify strong rules between the variables based on different measures of significance.

Association rule mining is an inference expression of the form $\mathrm{A} \rightarrow \mathrm{B}$ where $\mathrm{A}$ and $\mathrm{B}$ are two separate item sets in which $\mathrm{A} \cap \mathrm{B}=\varnothing$. Association rules are if-then statements that illustrate the probability of relationship between the items in a data base. It produces relevant relations between the variables in the form of association rules.[11]. Generation of rules is a two-step process. In the first step generation of frequent item set will be done by the system. The basic measure need to generate frequent item set is support count that includes number of times the items present in total transaction. Support

count of item sets more than the minsup where minsup is threshold value for support is considered as frequent item set.

Let $I=\{i 1, i 2, i 3, \ldots$, in $\}$ be a set of $n$ attributes called items and $D=\{t 1, t 2, \ldots, t n\}$ be the set of transactions. It is called database. Every transaction, ti in $\mathrm{D}$ has a unique transaction ID, and it consists of a subset of item sets in I.[17] $\mathrm{A}$ rule can be defined as implication, $A->B$ where $A$ and $B$ are subsets of $I(A, B \subseteq I)$, and they have no element in common, i.e., $\mathrm{A} \cap \mathrm{B}$. $\mathrm{A}$ and $\mathrm{B}$ are the antecedent and the consequent of the rule, respectively.[17]. There are numerous rules conceivable from a minor database, to select the interesting rules three different measures support, confidence, lift is castoff.

\section{Support:}

The support of an item set $\mathrm{A}, \sup (\mathrm{A})$ is the proportion of transaction in the database in which the item $\mathrm{A}$ appears. It signifies the popularity of an item set.[17]

$\operatorname{Supp}(A)=\frac{\text { Nopf trangation A appars }}{\text { Totai number of transactipons }}(1)$

\section{Confidence:}

Confidence for a rule is given as follows[17] 
$\operatorname{Conf}(A \rightarrow B)=\frac{\operatorname{sug}(A u B)}{\operatorname{sugp}(A)}$

Lift

Lift of a rule is given by[17]

$$
\operatorname{Lift}(A \rightarrow B) \frac{\text { Sugp }(A \cup B)}{\text { Supp }(A)+\operatorname{Supp}\left(B_{)}\right)}
$$

Support and confidence are two important measures for finding the interestingness of rules. These two measures replicate efficacy and certainty of the derived rule. Frequent item sets are those that have support value more than the minsup threshold value and crated as the first step of association rule mining in which $\mathrm{K}$ item sets present followed by deriving strong rules with minconf as threshold value.

\section{PROPOSED CULTURAL-BASED ASSOCIATION RULE GENERATION (CBARG) METHOD}

The idea behind the proposed technique of CBARG is to generate optimized rules based on evolutionary algorithm. Here the optimization has done in single phase instead of done in two phases by achieving parallelism. Parallelization improves performance of the algorithm.[24] Normally larger number of rules are generated by apriori algorithm which causes heavy computational time [5] since to produce the $\mathrm{L}_{k}$ ,algorithm has to scan the data base many times for generation of C1-k and item set which cannot be used to generate $L_{k}$ to be pruned and thus increase the time complexity as well as memory storage.[21] To find out the result appropriately though it satisfies minimum support and minimum confidence and lift values. In this proposed method once the individuals are created and evaluated with min sup and min confidence fitness value undergo the evolutionary process defined in the following sections and optimized rules are generated. The optimization system perfectly configurable for any changes[25]. Thus dropping the overhead cost of processing individuals with low fit values. CA [14] [15] is an adaptive mutation technique performing a heuristic search, inspired by the evolution process of genetics with the belief space. The basic conception of the evolutionary algorithm lies in its adaptive nature, it can change or fit itself according to the changing surroundings.[16] A population, containing the challenging results, is preserved, which experiences selection, crossover, and transformation to advance and congregate to the finest solution. A parallel search is achieved on the solution space to discover an optimal solution without getting trapped in a confined optimum. Cultural algorithm is an evolutionary algorithm and it's mechanism is based on the strategy survival of fittest and inspired by social evolution occurring in nature [15]. The original cultural algorithm has comprised of five knowledge sources in , belief space, population space and the communication protocols to exchange knowledge between the macro and micro level spaces. [15]

\subsection{Belief Space}

The belief space encompasses with five knowledge sources viz, the Normative, Situational, Domain, Topographical, and History knowledge source. [15][16]. All these knowledge sources are used to hold different knowledge congregated during the process of evolution which will be useful in solving the problem. This paper suggests the modified CA where the topographical knowledge source is not used for the heuristic search and new KS has been added.

\subsubsection{Normative Knowledge}

This knowledge source is used to store desirable range of values namely maximum and minimum list of possible values for the attributes define the population. These values are collected during the generation of rules and can be used during the cross over and mutation process.

\subsubsection{Situational Knowledge}

Situational knowledge source stores the best instances found during the evolution process. At the end of each generation this knowledge source is updated with this exemplar and is convenient for the evolution process to search for similar pattern of the same example instead of selecting blind random population.

\subsubsection{Domain Knowledge}

Domain knowledge source stores knowledge about problem province, domain objects. For this problem metric values for each rule generated and fitness values are stored to evaluate each rules produced and updated this knowledge source at the end of each generation. Here pareto optimality search principle is used by comparing the fitness values of each rule to select elite individual after each generation.

\subsubsection{History Knowledge}

History knowledge source archives temporal patterns of the searching process. In this study of research it stores dominant individual generated at the end of each generation by comparing the fitness values stored in domain knowledge source. Since the evolutionary algorithm is conceivable with memory contributed by these knowledge sources. But in general EAs are characterized as memory less as it does not store details about the previous generation which can be progressively changed the scenario and acquire memory in a systematic way with these different knowledge sources. Thus this history knowledge source stores elite population generated at each generation and maintain memory across the generations.

\subsubsection{Association Rule (AR) Knowledge Source}

This new knowledge source encompass the individuals produced during evolution using the optimized rule in (AR) knowledge source. The depiction of new knowledge source is similar to history knowledge source. This also render memory to evolutionary algorithm by retain elite individuals across the 
generations along with new optimized rules are added to new knowledge source at the end of each generations.

\section{Proposed Cultural-based Association Rule GENERATION (CBARG) METHOD FOR GENERATING THE OPTIMAL RULES FOR FINDING THE ASD.}

The Cultural Algorithm is the principle cycle in the proposed CBARG technique. Its parameters are the probabilities of mutation, crossover and Selection, thus achieve multi objective optimization. The best population is stored in the belief space of CA and the best population is returned by this proposed CBARG strategy. The initialization function is utilized to instate and to produce the best population. The function of output restores the best population if the end condition returns genuine. Something else, the parent population is chosen and the selection function is executed on the population. The population of offspring is delivered by performing the crossover function on the parent's population. In following stage, on some posterity chromosomes, the mutation function is executed, and the chosen of last population takes place among the populations of offspring and become parent for next generation

\subsection{Cultural-based Association Rule Generation Method Step by Step procedure}

Sep1:Input $\leftarrow$ Output of SU-FS from preprocessing (6)

Step 1.1:Generate Frequent Item set

Step 1.2: Encode each item set

Step 2: Population CBARG(sp,cp,mp)

Step 3: begin

Step 4: 1 - 0;

Step 5: pop[j] $\leftarrow$ initialize () ;

Step 6 while not terminate (pop[j]) do

Step 7: begin

Step 8: parents-pop $-\varnothing$

Step 9: childs-pop $-\varnothing$

Step 10:Evaluate fitness function for each set

Step 11: parents-pop -select(pop[j],sp)

Step 12: childs-pop-crossover (parents-pop,cp)

Step 13: childs-pop $\leftarrow$ mutate (childs-pop,cp)

Step 14: best-pop - pop[j]

Step 15Update Belief space

Step 16:Update Population space

Repeat step 5

Step 17: end

Stage 1: Encoding

THE list of data records are converted into chromosome called genotype consists of gene attributes. Basically cautious encoding method is chosen based on problem and the attributes value. In this study binary encoding is done. At this point $\mathrm{A} \rightarrow \mathrm{B}$ rule is antecedent and consequent is encoded using binary encoding consisting of bit strings.

\section{Stage 2: Initialization}

Initialization of population is an essential facet that decides the overall performance of the process. Here the initial chromosomes of the population are randomly generated. The ideal population is stored utilizing information space of CA.
Normative information is utilized in this work. The function of acceptance and influence is used for tolerating the belief space among the other generation.

Stage 3: Initialization of Fitness Function

The fitness function of CBARG method characterized is appeared in the below given Equation:

FF $=\frac{(1+\sup (\text { AuBDS }}{1+\text { Sup }(A)}$

The initial population is evaluated to find the fitness value of individuals. The select (pop,sp) goes about as a channel of chromosomes with the contemplations of their selection and fitness probability.

\section{Stage 4: Population selection}

In this phase the overall fitness of the population to be enhanced constantly. In this proposed technique, Tournament Selection 2 system is utilized so as to choose a specific number of chromosomes are chosen in premise of their fitness and then the best chromosome from them is seen in next population. This selection process discard the un fit individuals and keep the fittest individuals to produce descendants for next generation.

\section{Stage 5: Crossover Operator}

Crossover is a genetic operator castoff to combine part of their genomes to reproduce new progeny. Two individuals are taken selected in the selection phase and ready for cross over mating. Different cross over operators are suggested by many researchers[16][17][18]. In this proposed CBARG technique, the two-point crossover operator is utilized.

\section{Stage 6: Mutation Operator}

This operator works on individual values of chromosome. In this proposed CBARG technique, initial, random whole number from 1 to $\mathrm{i}-1$ that isolates the forerunner from consequence is created and relegated to first quality of chromosome, at that point, randomly, one quality is chosen from normative knowledge and its worth is changed. These minimum to maximum size of individuals of chromosome retrieved from Normative knowledge source.

\subsection{CBARG Frame work}

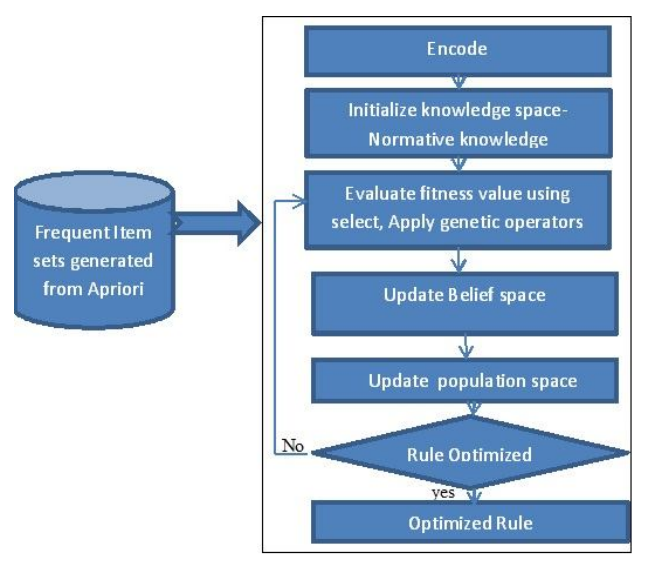

Figure 1 :CBARG Frame work 


\section{RESULT AND DISCUSSION}

\subsection{Description of the ASD Dataset}

The unique Autism Spectrum Disorder dataset is comprised of 75 features and optimal dataset has attained by using proposed cultural algorithm based feature selection method[20]. The optimal dataset is encompassed only 42 features by using proposed Cultural Algorithm based Feature Selection (SU-FS)[20]

\subsection{Measurement of Number of Rules Generated}

In the proposed CBARG method, the number of rules is created on the support and confidence values for different algorithms. It is clear if the number of rules is low, the technique is said to be more competent. Following Table 2 represents the number of rules produced by various supports and confidence values for different algorithms.

Table 1: Number of Rules generated by ARM, ARM-PSO, CA CBARG

\begin{tabular}{|c|c|c|c|c|}
\hline $\begin{array}{l}\text { SUPPORT } \\
\text { AND } \\
\text { CONFIDENCE }\end{array}$ & ARM & ARM-PSO & CA & $\begin{array}{l}\text { CBAR } \\
\text { G }\end{array}$ \\
\hline 0.2 and 0.2 & 3861 & 3412 & 3421 & 2741 \\
\hline 0.4 and 0.4 & 3631 & 3310 & 3201 & 2541 \\
\hline 0.6 and 0.6 & 2861 & 2421 & 2512 & 1862 \\
\hline 0.8 and 0.8 & 1893 & 1854 & 1745 & 1543 \\
\hline 1.0 and 1.0 & 967 & 948 & 845 & 423 \\
\hline
\end{tabular}

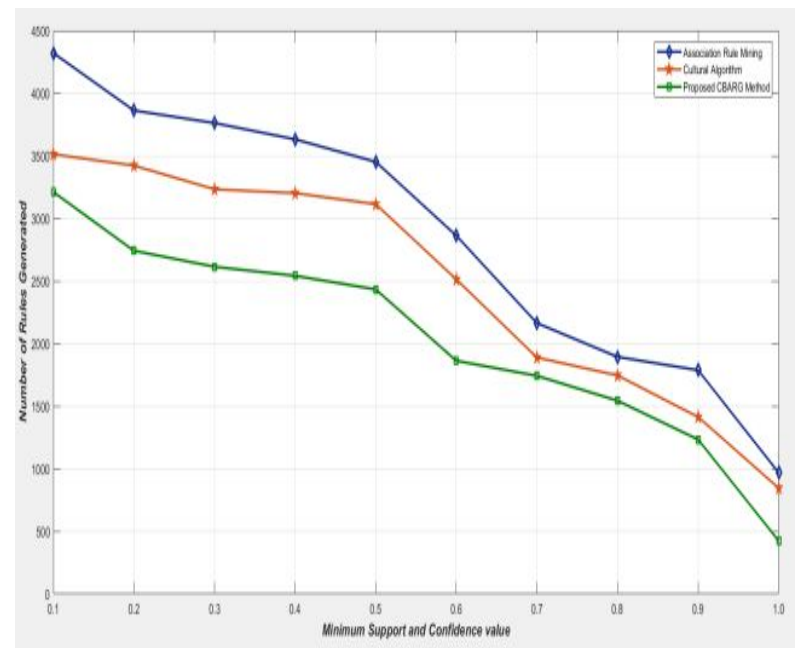

Figure 2: Performance analysis of Proposed CBARG, CA ARM and ARM-PSO in terms of number of rules generated against different values of support and confidence

\subsection{Memory Consumption for Rules generated}

Memory consumption denotes to the amount of memory taken for engendering the association rule, which is measured in terms of Mega Bytes (MB) [13].

$$
\mathrm{M} \text { (Memory Consumption) }=\mathrm{n} \times \mathrm{M}(\mathrm{n})
$$

where $\mathrm{M}$ is the memory consumption for association rule generation, $\mathrm{n}$ is the number of frequent generated rules, and
$\mathrm{M}(\mathrm{n})$ is the memory essential for generation of rules. When the memory consumption for association rule generation is low, the scheme is said to be more effective. Table 5 displays the memory consumption of the ARM, CA and proposed CBARG method by dissimilar support and confidence values.

Table 2: Memory Consumption(MB) for generated rules by using ARM, CA CBARG

\begin{tabular}{|c|c|c|c|c|}
\hline $\begin{array}{l}\text { SUPPORT AND } \\
\text { CONFIDENCE }\end{array}$ & ARM & ARM-PSO & CA & $\begin{array}{l}\text { CBAR } \\
\text { G }\end{array}$ \\
\hline 0.2 and 0.2 & 34.1 & 32.1 & 33.4 & 30.3 \\
\hline 0.4 and 0.4 & 33.1 & 33.1 & 30.1 & 22.0 \\
\hline 0.6 and 0.6 & 29.5 & 26 & 24.2 & 19.5 \\
\hline 0.8 and 0.8 & 19.4 & 18 & 18.5 & 13.9 \\
\hline 1.0 and 1.0 & 9.5 & 8 & 7.8 & 3.1 \\
\hline
\end{tabular}

Figure 3: Performance analysis of Proposed CBARG, CA ,ARM-PSO and ARM in terms of memory consumption against different values of support and confidence

\subsection{Algorithm Complexity}

The computational time for frequent item set generation processes the amount of time taken for producing the frequent item sets with reverence to given support and confidence values. It is weighed in terms of milliseconds (ms) and arithmetically formulated as follows [13],

$$
\mathrm{RT}=\mathrm{n} \times \mathrm{T}(\mathrm{n})
$$

where RT is the running time, $\mathrm{n}$ signifies the number of frequent item sets generated, and $\mathrm{T}(\mathrm{n})$ denoted time taken for frequent item set generations. Once the running time for frequent item set generation is low, the method is said to be more competent.

Table 3: Total running time(ms) of ARM, ARM-PSO,CA and CBARG

\begin{tabular}{|c|c|c|c|c|}
\hline $\begin{array}{l}\text { SUPPORT AND } \\
\text { CONFIDENCE }\end{array}$ & ARM & ARM-PSO & CA & CBARG \\
\hline 0.2 and 0.2 & 301 & 341 & 332 & 224 \\
\hline 0.4 and 0.4 & 278 & 331 & 285 & 220 \\
\hline 0.6 and 0.6 & 205 & 242 & 242 & 196 \\
\hline 0.8 and 0.8 & 176 & 185 & 194 & 165 \\
\hline 1.0 and 1.0 & 71 & 78 & 95 & 33 \\
\hline
\end{tabular}




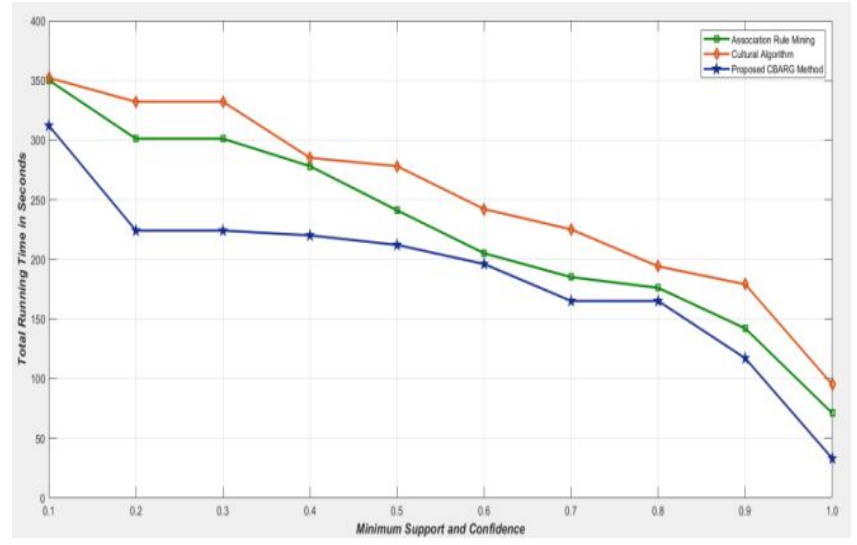

Figure 4: Performance analysis of Proposed CBARG , CA,ARM-PSO and ARM in terms of Computation time (in Seconds) against different values of support and confidence

From this above tables, it has comprehended that projected CBARG method runs speedily than the other algorithms in maximum case.

\section{CONCLUSION}

The autism spectrum disorders belong to an "umbrella" class category of five childhood-onset conditions known as pervasive developmental disorders (PDD). Detecting the patterns for ASD is grown as more thought-provoking in factual world for learning persistence. In this paper, a new method called Cultural-based Association Rule Generation (CBARG) algorithm established on Apriori Rule Mining (ARM) and Cultural Algorithm (CA) to find out the pattern belongs to ASD category by generating strong rules to strengthen the ASD yes category variable with lesser number of rules, computational time and memory consumption. As the rules which are not able to compete with high support and fitness value rules are eliminated by the CBARG and produce optimized rules, it is observed proposed CBARG contributes more in predicting the results. The frequent item set are retained and incorporated into the fitness function of $\mathrm{CA}$. The experiment is done by using ASD dataset asserts that the number of rules, memory consumption and computation time of the proposed CBARG method are superior than those of the combining methods ARM and CA.

\section{REFERENCES}

1. Jerry, C. W. L., Wensheng G., Philippe F.-V., Tzung-P. H., Vincent S. T. Fast algorithms for mining high-utility itemsets with various discount strategies, Advanced Engineering Informatics, 30(2), 109-126, 2016. https://doi.org/10.1016/j.aei.2016.02.003

2. Thabtah, F., Qabajeh, I., \& Chiclana, F. Constrained dynamic rule induction learning, Expert Systems with Applications, 63, 74-85, 2016. https://doi.org/10.1016/j.eswa.2016.06.041

3. C.X. Chen, J.J. Shen, B. Chen, C.X. Shang, Y.C. Wang. An improvement apriori arithmetic based on rough set theory, Third Pacific-Asia Conference on Circuits,
Communications and System (PACCS) IEEE, pp. 1-3, 2011.

4. Martínez-Ballesteros M., Bacardit, J., Troncoso A., \& Riquelme, J. C. Enhancing the scalability of a genetic algorithm to discover quantitative association rules in large-scale datasets. Integrated Computer-Aided Engineering, 22(1), 21-39, 2015.

5. Mai,T., Vo,B., Nquyen, L.T. A Lattice-based approach for mining high utility association rules. Information Sciences, 399,81-97, 2017.

6. Abitha R,Mary Vennila S. A proficient optimized feature selection method germane for Autism Spectrum Disorder Classification. International journal of Scientific and Technology Research (under printing)

7. Song, A., Ding, X., Chen, J., Li, M., Cao, W., \& Pu, K.. Multi-objective association rule mining with binary bat algorithm. Intelligent Data Analysis, 20(1) 105-128,2016.

8. Uros Mlakar, Milan Zorman, Iztok Fister Jr, Iztok Fister. Modified binary cuckoo search for association rule mining. Journal of Intelligent \& Fuzzy Systems (Preprint), 1-12.IOS press, 2017.

9. Yan, C., Sun, H., \& Liu, W. Study of fuzzy association rules and cross-selling toward property insurance customers based on FARMA. Journal of Intelligent \& Fuzzy Systems, 31(6), 2789-2794,2016.

10. Shirsath, P. A., \& Verma, V. K.. A Recent Survey on Incremental Temporal Association Rule Mining. International Journal of Innovative Technology and Exploring Engineering, 3(1), 85-92,2013.

11. Yan, Xuesong, Wei li, Qinghua Wu,Victor S. Sheng. A double weighted Naive Bayes for multi-label classification. International Symposium on Computational Intelligence and Intelligent Systems. Springer, 382-389,2015.

12. Kerin, Tara, Heather Volk, et al. Association Between Air Pollution Exposure, Cognitive and Adaptive Function, and ASD Severity Among Children with Autism Spectrum Disorder. Journal of autism and developmental disorders 48.1, 137-150, 2018.

13. Sathya M \& Thangadurai K. Association Rule Generation Using E-ACO Algorithm. International Journal of Control Theory and Applications, 27(9), 513-521, 2016.

14. Le Hoang Son, Francisco Chiclana, Raghavendra Kumar, Mamta Mittal, Manju Khari,Jyotir Moy Chatterjee, Sung Wook Baik. ARM-AMO : An efficient association rule mining algorithm based on animal migration optimization. Knowledge -Based Systems Vol 154, 68-80, Aug 2018. https://doi.org/10.1016/j.knosys.2018.04.038

15. Reynolds, Robert G. The cultural algorithm: Culture on the edge of chaos. Culture on the edge of chaos. Springer, Cham, 1-11,2018.

16. Muhamad Kamal Amjad, Shahid Ikramullah Butt,Rubeena Kousar,Riaz Mohamed. Recent Research 
Trendsin Genetic Algorithm Based Flexible Job Shop Sheduling Problems. Mathematical Problems in Engineering. ID 9270802,32, volume 2018.

17. https://www.hackerearth.com

18. N.S. Nithya, K. Duraiswamy. Correlated gain ratio based fuzzy weighted association rule mining classifier for diagnosis health care data. Journal of Intelligent and Systems, 29 (4) 1453-1464, 2015.

DOI: $10.3233 /$ ifs-151614

19. Kargarfard, F., A. Sami, and E. Ebrahimie, Knowledge discovery and sequence -based prediction of pandemic influenza using an integrated classification and association rule mining (CBA) algorithm. Journal of Biomedical Informatics, 57:181-188, 2015.

20. R.Abitha., Dr. S.Mary Vennila. A Swarm Based Symmetrical Uncertainty Feature Selection Method For Autism Spectrum Disorders. $3^{\text {rd }}$ International Conference on Inventive Systems and Control. IEEE, 2019.

21. Grami,M., Ghebi, R.,Rahimi, F. A novel Association Rule Mining Using Genetic Algorithm. Eigth International Conference on Information and Knowledge Technology(IKT), IEEE ,2016.

22. Djenouri, Y., Drias, H. and Habbas, Z. Bees swarm optimisation using multiple strategies for association rule mining. International Journal of Bio-Inspired Computation, Vol. 6, No. 4, pp.239-249,2014.

23. Jia Wang ,Hongguang Li, Jingwen Huang, Chong Su. Association rules mining based analysis of consequential alarm sequences in chemical processes. Journal of Loss Prevention in the Process Industries.41(2016): p. 178-185.

24. Maria Zemzami, Aicha Koulou, Norelislam Elhami, Mhamed Itmi,Nabil Hmina. An evolutionary hybrid algorithm for complex optimization problem. International Journal of Advanced Trends in Computer Science and Engineering. Vol.8, No.2, March-April 2019. https://doi.org/10.30534/ijatcse/2019/05822019

25. MariaZemzami,AichaKoulou, Norelislam Elhami, Mhamed Itmi,Nabil Hmina. Interoperability Optimization using a modified PSO algorithm. International Journal of Advanced Trends in Computer Science and Engineering. Vol.8, No.2, March-April 2019. https://doi.org/10.30534/ijatcse/2019/01822019

26. Mohit K. Gupta., Geeta Sikka. Association Rules Extraction using Multi-Objective Feature of Genetic Algorithm. Proceedings of the World Congress on Engineering and Computer Science 2013 Vol II WCECS 2013, 23-25 October, 2013, San Francisco, USA 